\title{
A Joint Evaluation of Different Dimensionality Reduction Techniques, Fusion and Learning Methods for Action Recognition ${ }^{\text {th }}$
}

\author{
Haiyan $\mathrm{Xu}^{\mathrm{a}}$, Qian Tian ${ }^{\mathrm{a}}$, Zhen Wang ${ }^{\mathrm{a}}$, Jianhui $\mathrm{Wu}^{\mathrm{a}, *}$ \\ ${ }^{a}$ National ASIC System Engineering Research Center, Southeast University, Naijing, \\ Jiangsu 210096, China.
}

\begin{abstract}
This paper addresses the problem of action recognition with improved dense trajectories (IDT). Recently, IDT achieved a significant performance in action recognition with realistic videos. However, the efficiency of storage and the speed of classification are limited due to the dense samples in feature space. To address this issue, the intuitive way is to reduce the dimension and adopt a fast classification method. Therefore, we explore the influence of dimensionality reduction on the recognition rate. In addition, Extreme Learning Machine (ELM) is adopted to further improve classification efficiency. We present performance on the KTH, UCF11, HMDB51, and UCF101 datasets in all kinds of situations such as the different fusion methods, the different dimensionality reduction, and different learning methods. As a result, it can be observed that ELM with principal components analysis (PCA) improves the performance in terms of mean average precision (mAP) which not only significantly reduces computational cost but improves accuracy. What's more, the training and testing time decrease $1-2$ orders of magnitude without losing accuracy when Fisher vector $(\mathrm{FV})$ adopts reduction techniques before it fed into classifier.
\end{abstract}

Keywords: human action recognition; different fusion methods; different dimensionality reduction; different machine learning methods; video signal

\footnotetext{
${ }^{*}$ Corresponding author

Email address: wjh@seu.edu.cn (Jianhui Wu)
}

Preprint submitted to Journal of ${ }^{A} T_{E} X$ Templates

April 14, 2016

(C) 2016. This manuscript version is made available under the Elsevier user license http://www.elsevier.com/open-access/userlicense/1.0/ 
processing;

\section{Introduction}

Action recognition has caught the attention of researchers from real-time video surveillance and security monitoring, automatic video indexing, and humancomputer interfaces. Meanwhile, the problem of action recognition in videos is challenging, and all difficulties associated with object detection and recognition task, such as large intra-class variations, low resolution, camera motion, view point changes, geometric and photometric variances, may also be encountered in action recognition.

In recent years, dense sampling has great potential in improving recognition rate over sparse interest points [1. Wang et al. 2] 3] 4] proposed an approach to describe videos by dense trajectories (DT) which has a significant improvement over the state of the art on some realistic data sets with fisher vector $(\mathrm{FV})$. However, the DT based representation is expensive in memory storage and computation due to the large number of densely sampled points. What's more, they need to combine all the descriptors which are histogram of oriented gradients (HOG), histogram of optical flow (HOF), motion boundary history $(\mathrm{MBH})$ and trajectory (Dentr) to obtain the best results, and the efficiency is also a problem for the dense trajectories and FV dimension. Hence, Murthy et.al. 5] proposed to select only a few dense trajectories and evaluate it on the complex dataset with improved performance. In this paper, we apply principal component analysis (PCA) or linear discriminant analysis (LDA) to reduce the dimension for local descriptor and video-level vector (i.e., FV in this paper), respectively. Finally, we evaluate all kinds of fusion methods which fuse those descriptors by probability fusion, video-level fusion or local feature fusion.

As local features' discriminative power is low and its data is large which limit the efficiency and memory usage, it is difficult to directly classify the local features (i.e., low-level representation). Hence, three main vector aggregation methods (i.e., Bag of features, Fisher Vector,VLAD) are used for action recog- 
nition [6]. Bag of features (BOF) is popularly used for aggregating local cuboid descriptors into compact codes [2] [7] 8] 9]. However, it only encodes the zero statistics information and lacks structural information. Therefore, the fisher kernel (i.e., fisher vector) is used for large-scale image classification [10] and action recognition [3]. Fisher vector (FV) encodes both first and second statistics between the video descriptors and a Gaussian Mixture Model (GMM). Vector of locally aggregated descriptors (VLAD) was first proposed by Jegou in [11] for image search and index which was thought as a simplification of the fisher vector. This technique was first considered by Jain et al. [12] for action recognition. In paper [10] it shows that the fisher vector was more suitable than BOF for largescale image classification, which achieves better results for action recognition in

40 3]. In addition, FV only needs to use a linear SVM for classification which is efficient. Therefore, FV is used in our paper. However, for each descriptor, a 2DK dimensional FV is used to represent it. Where $\mathrm{D}$ is the descriptor dimension, $\mathrm{K}$ is the number of Gaussian Mixture Model (GMM). To better fit FV encoding, PCA is used to reduce the descriptor dimensionality. Normally, the value of $\mathrm{K}$ is set to be 256 for action recognition to ensure the performance and efficiency [3] [5], however, 512D it is still large for each descriptor's FV. Therefore, before FV feeds into classifier, dimensionality reduction technique can be applied to FV to improve efficiency and save storage. In this paper, we evaluate the influence of different dimensionality reduction technique in terms of mean average precision (mAP) for action recognition. From the experiment, it shows that FV adopts dimensionality reduction which can guarantee or improve the performance and efficiency.

In action recognition for different vector-level representations, classifier mainly uses $\chi^{2}$ kernel or linear support vector machine (SVM) 2, 13] 14] 15] [16]. For large-scale datasets, due to the increase number of training samples, the efficiency of SVM will decrease. Therefore, in our paper, we will use the Extreme Learning Machine (ELM) for action classification. The fast and efficient ELM was used by Iosifideis et.al [17, Minhas et.al[18 and Varol et.al[19] for action recognition, but the usage of ELM in the action recognition area is still rare. 
Hence, in our paper, we not only uses ELM but compares it with SVM for action recognition.

This paper is organized as following: in section II, we analyze the related work. We establish the framework and expatiate our method for action recognition in section III. The experimental results are presented in Section IV. Finally,

65 we make a conclusion of this paper in section $\mathrm{V}$.

\section{Related work}

Various work has studied the problem of based-video human action recognition. In this section, we give an overview of previous work which is most relevant to our proposed method.

Improved dense trajectories (IDT) 3] 20] 21] was popularly used for action recognition with realistic videos on account of significant performance. Due to the large number of densely sampled points, the IDT based representation is expensive in memory storage and computation. Therefore, it is important to improve the efficiency of memory storage and computation. Shi et al. [22] 23] 75 adopted PCA to local feature descriptor for better fit FV encoding. Ikizler et al. 24] first applied LDA to obtain a more compact and discriminative feature representation. These methods have been proved quite successful to reduce the dimension of local feature that is better for FV encoding. LDA is a popular method for extracting features that preserves class separability. However it is expensive in memory usage and computation. Hence, Cai's 25] LDA which is called Spctral Regression Discriminative Analysis (SRDA) is used in our paper. SRDA only needs to solve a set of regularized least squares problems, and there is no eigenvector computation involved, which saves both time and memory highly. FV's dimension is $2 \mathrm{DK}$ which is larger than the BOW. In this paper, we concentrate on how to reduce the dimension of FV before it feeds into classifier.

Although only linear SVM classifier is needed to classify FV, it is expensive for large-scale dataset such as HMDB51 [26], UCF101 [27]. Therefore, a faster classifier is used in this paper to improve the efficiency. ELM was used for 
action recognition in recent years [17] 18] 19]. Iosifidis et al. [17] proposed a discriminant ELM algorithm to exploit information appearing in both labeled and unlabeled action instances. Minhas et al. [18, and Varol et al. [19] used ELM for action recognition with large-scale dataset. Though ELM is fast and efficient, it is rarely used for action recognition. Thus, in this paper, we explore its efficiency and performance for action recognition.

\section{Our framework}

In this paper, we discuss our framework in three parts. First, we introduce the basic flow chart (see Fig.1). Second, we discuss the different fusion methods used in this paper which include local feature fusion, mid-level fusion, and classifier results fusion (called the late fusion). And the first two fusion we generally call it early fusion. Third, we briefly introduce the machine learning methods which are used in our paper.

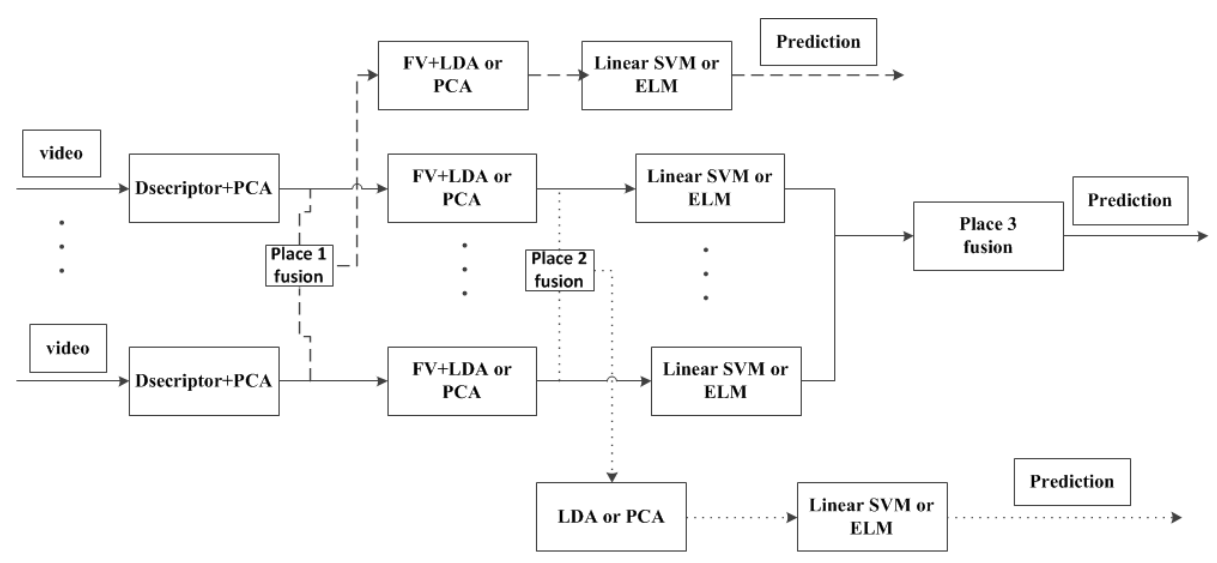

Figure 1: The data-flow for our action recognition system

\subsection{The basic flow chart for action recognition}

\subsubsection{Local feature descriptors of dense trajectories}

In this paper, we use the low-level representation which was proposed by

Wang [3]. For those descriptors, we use PCA to reduce the number of features 
such as HOG,HOF,MBH and Dentr which is the trajectory descriptor proposed in paper 3 for better fit FV encoding. The data-flow is shown in Fig.1.

First, dense trajectories are extracted from eight spatial scales. Feature points are sampled on a grid spaced by $\mathrm{W}$ pixels and tracked in each scale sep-

arately. Then, the sampled points are tracked by applying median filtering over the dense optical field. The MBH, HOG, HOF and Dentr were computed on the neighbourhood of the points derived by the dense trajectories. In our experiment, we use the default $\mathrm{W}=5$ pixels. The work in 3 . shows that the space-time volumes (spatial size $32 \times 32$ pixels) around the trajectories are divided into 12 equal-sized $3 \mathrm{D}$ grids(spatially, $2 \times 2$ grids and temporally, 3 segments). For both HOG and HOF, orientations are quantized into 8 bins using full orientations, with an additional zero bin for $\mathrm{HOF}$ (i.e., in total 9 bins). MBH are computed separately for the horizontal and vertical components of the optical flow. Thus, the HOG descriptors have 96 dimensions, HOF descriptors have 108 dimensions and $\mathrm{MBH}$ descriptors have 192 dimensions. For $\mathrm{L}=15$ which is the trajectory of given length, the trajectory obtained a 30 dimensions. In order to improve memory storage and computation, we apply PCA for those descriptors which is reduced to $64,64,128,20$, respectively for place 2 and place3 fusion. While for place1 fusion we combined the local features together that have 426 dimensions.

${ }_{225}$ In this situation, we apply PCA for it to reduce to 200. What's more, PCA can improve performance by decorrelated data.

\subsubsection{Aggregating method}

As we know, Fisher vector (FV) was popularly used for action recognition in recent years 528 16 29. Because FV representation has a lot of advantages, such as training fast and improving the accuracy for large-scale action recognition. Therefore, we adopt FV aggregating methods in this paper.

We directly aggregate HOG, HOF, MBH and Dentr by Fisher kernel, to obtain the FV. The Fisher kernel (FK) is a generic framework which combines the benefits of generative and discriminative approaches. The FK produces fisher vector which is the gradient of the sample's likelihood with respect to the 
parameters of this distribution. Let $v=\left(X_{1} \ldots X_{N}\right)$ be a set of D dimensional features vectors (e.g. HOG, HOF, MBH, etc.) extracted from a video. Let $\Theta=\left(\mu_{k}, \Sigma_{k}, \Pi_{k}: k=1, \ldots, K\right)$ be the parameters of a Gaussian Mixture Model (GMM) fitting the distribution of descriptors, among which $\mu_{k}, \Sigma_{k}$, and $\Pi_{k}$ are respectively the mean vector, covariance matrix and mixture weight. We assume that the covariance matrices are diagonal and denote by $\sigma_{k}^{2}$ the variance vector. The GMM associates each vector $X_{i}$ to a mode $\mathrm{k}$ in the mixture with a strength given by the posterior probability:

$$
q_{i k}=\frac{\exp \left[-\frac{1}{2}\left(X_{i}-\mu_{k}\right)^{T} \Sigma_{k}^{-1}\left(X_{i}-\mu_{k}\right)\right]}{\Sigma_{t=1}^{K} \exp \left[-\frac{1}{2}\left(X_{i}-\mu_{k}\right)^{T} \Sigma_{t}^{-1}\left(X_{i}-\mu_{k}\right)\right]}
$$

For each mode $\mathrm{k}$, considering the mean and covariance deviation vectors

$$
\begin{gathered}
u_{j k}=\frac{1}{N \sqrt{\Pi_{k}}} \sum_{i=1}^{N} q_{i k} \frac{X_{j i}-\mu_{i k}}{\sigma_{i}} \\
v_{j k}=\frac{1}{N \sqrt{\Pi_{k}}} \sum_{i=1}^{N} q_{i k}\left[\left(\frac{X_{j i}-\mu_{i k}}{\sigma_{i}}\right)^{2}-1\right]
\end{gathered}
$$

where $j=1,2, \ldots, D$ spans the vector dimensions. The $\mathrm{FV}$ of videos $v$ is the stacking of the vectors $u_{k}$ and the vectors $v_{k}$ for each of the $\mathrm{K}$ models in the Gaussian mixtures:

$$
\Phi(V)=\left[\ldots u_{k} \ldots v_{k} \ldots\right]^{T}
$$

where $[\bullet]^{T}$ means the transpose of vector. Hence, each video is represented by a 2DK dimensional FV for each descriptor type. Before being fed into classifier, we use FV by applying the signed square-rooting and following with L2 normalization.

The work in [30] shows that the mAP increases with the $\mathrm{K}$ for image search. However, for the high value of $K$, the dimensionality of the fisher vector becomes very large and is not suitable for high efficiency. Therefore, we apply LDA or PCA technique before FV is fed into classifier. Finally, linear classifiers are used to classify the action. 


\subsection{Different fusion methods}

As we know, single feature only emphasises on a part of aspects of actions or

155 and $\mathrm{HOF}$ which captures the motion information. Hence, It is an important issue to know how to combine them. Marszalek et al. [15] combined HOG $\backslash \mathrm{HOF}$ to increase recognition rate for Hollywood1 dataset. Early fusion was popularly used for action recognition in 31 32 33 34 35. Wh. While late fusion research is rarely considered for action recognition in recent years. In this paper, we apply different fusion methods to improve the recognition rate and compare them.

For each descriptor, early fusion and late fusion are respectively used to combine them. And, there are two places to fuse the descriptor for early fusion, which are place1 (local feature fusion) and place2 (video-level fusion) fusion, respectively, as shown in Fig.1. Late fusion is corresponding to place3 in Fig.1. Meanwhile, in [16], which are also namely descriptor-level fusion, kernel-level fusion and score-level fusion.

Place1 fusion combines the Space-Time interest points (STIP) features (see the Dotted arrows of the Fig.1 after the box of place1 fusion); place2 fusion combines FV representations (see the point arrows of the Fig.1 after the box of place 2 fusion). Before being fed into classifier, the combination of $\mathrm{FV}$ further adopt LDA or PCA to reduce the dimension. And place3 fusion combines the output of classifiers. In this paper, we will evaluate the three fusion methods respectively, which shows that place2 fusion will be better than place1 and place3 fusion except the case of ELM in UCF11.

\subsection{Different machine learning methods}

For each descriptor's FV, the linear classifier will be used to classify them which has low cost [3] 12. In our experiment, we set the regularization parameter by cross-validation. Two different linear classifiers (SVM and ELM) are adopted for descriptor's learning.

In machine learning, support vector machines are supervised learning models associated with learning algorithms that analyze data and recognize patterns, 
used for classification. For FV representations, linear SVM classifier is suitable for classification [10] [16] 28]. In our paper, first, we used the popular method which is SVM for FV representation. For probability fusion of late fusion, the training is performed with probability models, which provide the probability estimates for testing. From the experiment, it shows that the training and testing time (not including pretreatment time in our paper) are expensive for large-scale dataset, therefore, we try to use the ELM machine learning methods.

Extreme Learning Machine is proposed by Huang et al. as an extremely fast alternative to other conventional popular learning algorithms [36]. The proposed algorithm works for the generalized single-hidden-layer feed-forward networks, but the difference is that the hidden layer in ELM does not need to be tuned [37. 17] 18] 19] used ELM for action recognition. In our method, we use linear kernel ELM and the parameter was selected based on cross-validation on the training set of the different datasets.

In our paper, for late fusion of SVM, we explore the different modes to combine those classifiers which are trained by all kinds of descriptors. It has six rules to combine those classifiers which are sum, product, max, min, majority, median, respectively [29]. What's more, for ELM, we also use the same rules as SVM. The rules input is probability for SVM, while the scores as input for ELM. In the case of multi-class classification, we use a one-vs-one strategy.

\section{Experimental results and analysis}

In this section, we introduce the KTH, Youtube (UCF11), HMDB51 and UCF101 dataset. Then, we analyze our results and list confusion matrix of the best result. The first combination (Comb.1) is corresponding to HOG, HOF and $\mathrm{MBH}$ descriptor, and the second combination (ALL_combine) is corresponding to Dentr, HOG, HOF, and MBH. In the place1 fusion we use ALL_combine, while the Comb.1 and ALL_combine are all evaluated in the place2 fusion. For fast classification and to improve accuracy, we use LDA and PCA for FV representations, respectively in the place 2 fusion and the rate is fixed for $100 \%$. In the 


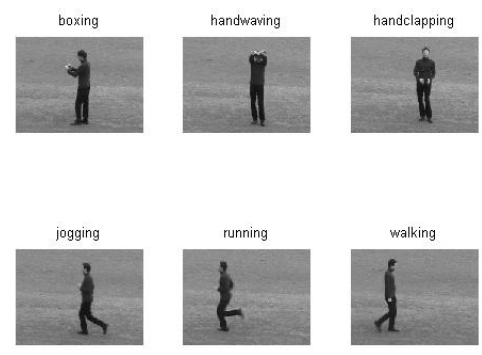

Figure 2: The type of actions on KTH 39]

place3 fusion only the Comb.1 is evaluated. Furthermore, the best performance among every table is shown in boldface.

The efficiency of dimensionality reduction techniques is only compared to UCF11 dataset. Because in constrained dataset the efficiency problem does not exist, and in large-scale dataset it is very time consuming and require good hardware condition without reduction techniques. For simplicity, in table 5 tr_time and te_time mean training and testing time respectively.

\subsection{Experimental setups} walking, jogging, boxing, handclapping and handwaving) in 4 different scenarios (indoors, outdoors, outdoors with scale changes and outdoors with different clothes). It contains 599 videos, of which 399 are used for training, and the rest for testing. It should contain 600 videos but one is missing. The evaluation condition is the same as 38.

The Youtube dataset [40] contains 11 (UCF11) action categories: biking cycling, diving, golf swinging, soccer juggling, trampoline jumping, horse riding, basketball shooting, volleyball spiking, swinging, tennis swinging, and walking with a dog (see Fig.3). This dataset is challenging due to large variations in camera motion, object appearance and pose, object scale, viewpoint, cluttered background and illumination conditions. The dataset contains a total of 1,168 


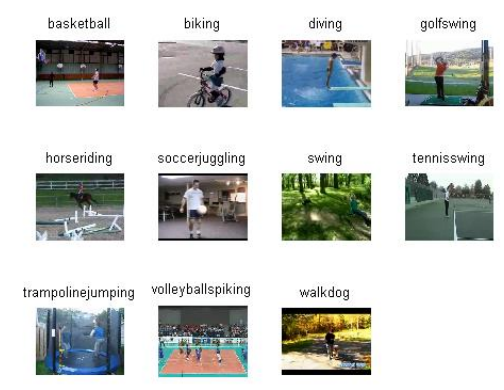

Figure 3: The type of actions on UCF11 40]

sequences. We follow the original setup using Leave-One-Out Cross-Validation for a pre-defined set of 25 groups. Average accuracy over all classes is reported as the performance measure.

HMDB51 [26] is compose of 6849 videos clips which are divided into 51 action categories. the action categories can be grouped in five types: 1) General facial actions (4): smile, laugh, chew, talk; 2) facial actions with object manipulation (3): smoke, eat, drink; 3) general body movements (19): cartwheel, clap hands, climb, climb stairs, dive, fall on the floor, backhand flip, handstand, jump, pull up, push up, run, sit down, sit up, somersault, stank up, turn, walk, wave; 4) body movements with object interaction (18): brush hair, catch, draw sword, dribble, golf, hit something, kick ball, pick, pour, push something, ride bike, ride horse, shoot ball, shoot bow, shoot gun, swing baseball bat, sword exercise, throw; 5) body movements for human interaction (7): fencing, hug, kick someone, kiss, punch, shake hands, sword fight. Some of the actions with this dataset see Fig.4. The different actions have large appearance variations. It all adopts the default training and testing splits [26].

UCF101 27] has 101 action classes. The action classes can be divided into five types: human object interaction, body-motion only, human-human interaction, playing musical instruments, and sports. Totally, it has 13,320 video clips. To the best knowledge, this dataset has been the largest dataset so far. We perform evaluation on the first split of UCF101 according to the default 


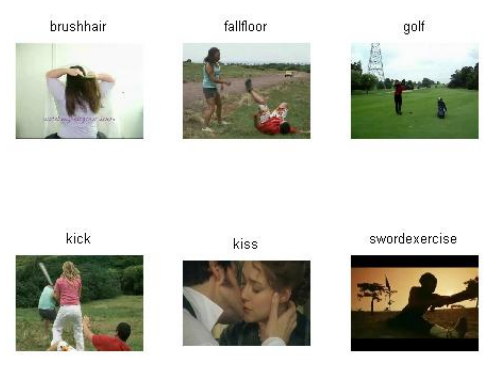

Figure 4: Some type of actions on HMDB51 [26]

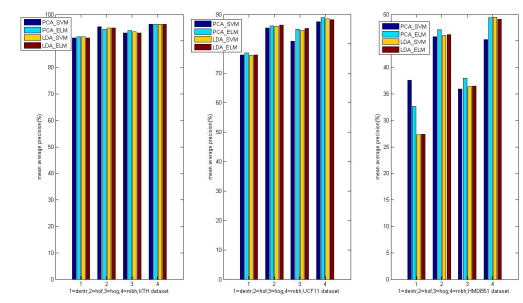

Figure 5: compare different reduction techniques and learning methods

training and testing splits.

\subsection{The performance of different dimensionality reduction techniques}

255

In this section, we evaluate the impact of LDA and PCA on mAP and training \testing time for video-level representation (in this paper, it is FV). Table 1 and table 2 shows that PCA has no impact on the mAP but it is fast for classification see table 6 (non_PCA means that there is no dimensionality reduction for FV). It shows that the efficiency of LDA or PCA can be increased 1-2 orders of magnitude compared to non_PCA with SVM on UCF11. Therefore, in order to save computation cost and memory while preserving the accuracy, PCA will be used to be compared with LDA in the subsection for different datasets, different fusion methods and learning methods in table 1-4.

Seen "yellow and blue bar" of fig.5, LDA with SVM achieved the similar or better performance than PCA with SVM except the Dentr's FV of HMDB51. 
Table 1: dimensionality reduction technique comparison on the KTH dataset with different learning methods and fusion methods

\begin{tabular}{|c|c|c|c|c|c|c|c|c|}
\hline learning methods $\rightarrow$ & \multicolumn{4}{|c|}{ SVM } & \multicolumn{4}{|c|}{ ELM } \\
\hline descriptor & \multicolumn{2}{|c|}{ LDA } & $\mathrm{PCA}$ & non_PCA & \multicolumn{2}{|c|}{ LDA } & \multicolumn{2}{|c|}{ PCA } \\
\hline Dentr & \multicolumn{2}{|c|}{$91.67 \%$} & $91.20 \%$ & $91.20 \%$ & \multicolumn{2}{|c|}{$91.20 \%$} & \multicolumn{2}{|c|}{$91.67 \%$} \\
\hline $\mathrm{HOF}$ & \multicolumn{2}{|c|}{$94.91 \%$} & $95.37 \%$ & $95.37 \%$ & \multicolumn{2}{|c|}{$94.91 \%$} & \multicolumn{2}{|c|}{$94.44 \%$} \\
\hline HOG & \multicolumn{2}{|c|}{$93.52 \%$} & $93.06 \%$ & $93.06 \%$ & \multicolumn{2}{|c|}{$93.06 \%$} & \multicolumn{2}{|c|}{$93.98 \%$} \\
\hline $\mathrm{MBH}$ & \multicolumn{2}{|c|}{$96.30 \%$} & $96.30 \%$ & $96.30 \%$ & \multicolumn{2}{|c|}{$96.30 \%$} & \multicolumn{2}{|c|}{$96.30 \%$} \\
\hline place1 fusion & \multicolumn{2}{|c|}{$96.76 \%$} & \multicolumn{2}{|c|}{$\mathbf{9 7 . 2 2} \%$} & \multicolumn{2}{|c|}{$\mathbf{9 6 . 7 6} \%$} & \multicolumn{2}{|c|}{$\mathbf{9 6 . 7 6} \%$} \\
\hline place2 fusion & LDA & $\mathrm{PCA}$ & LDA & PCA & LDA & $\mathrm{PCA}$ & LDA & $\mathrm{PCA}$ \\
\hline Comb.1 & $96.76 \%$ & $\mathbf{9 7 . 2 2} \%$ & $96.30 \%$ & $96.30 \%$ & $95.83 \%$ & $95.83 \%$ & $94.91 \%$ & $\mathbf{9 6 . 7 6} \%$ \\
\hline ALL_combine & $96.30 \%$ & $96.30 \%$ & $96.30 \%$ & $96.30 \%$ & $95.37 \%$ & $95.37 \%$ & $95.37 \%$ & $96.30 \%$ \\
\hline place3 fusion $\downarrow$ & \multicolumn{2}{|c|}{ LDA } & \multicolumn{2}{|c|}{ PCA } & \multicolumn{2}{|c|}{ LDA } & \multicolumn{2}{|c|}{ PCA } \\
\hline product & \multicolumn{2}{|c|}{$\mathbf{9 7 . 2 2} \%$} & \multicolumn{2}{|c|}{$96.76 \%$} & \multicolumn{2}{|c|}{$94.91 \%$} & \multicolumn{2}{|c|}{$92.59 \%$} \\
\hline sum & \multicolumn{2}{|c|}{$\mathbf{9 7 . 2 2} \%$} & \multicolumn{2}{|c|}{$96.76 \%$} & \multicolumn{2}{|c|}{$95.83 \%$} & \multicolumn{2}{|c|}{$\mathbf{9 6 . 7 6} \%$} \\
\hline $\max$ & \multicolumn{2}{|c|}{$96.76 \%$} & \multicolumn{2}{|c|}{$96.30 \%$} & \multicolumn{2}{|c|}{$95.83 \%$} & & $6 \%$ \\
\hline median & & $6 \%$ & & $30 \%$ & & $0 \%$ & & $6 \%$ \\
\hline $\min$ & & $6 \%$ & & $76 \%$ & & $1 \%$ & & $3 \%$ \\
\hline major & & & & $30 \%$ & & $0 \%$ & & $6 \%$ \\
\hline
\end{tabular}


Table 2: dimensionality reduction technique comparison on the UCF11 dataset with different learning methods and fusion methods

\begin{tabular}{|c|c|c|c|c|c|c|c|c|}
\hline learning methods $\rightarrow$ & \multicolumn{4}{|c|}{ SVM } & \multicolumn{4}{|c|}{ ELM } \\
\hline descriptor & \multicolumn{2}{|c|}{ LDA } & PCA & non_PCA & \multicolumn{2}{|c|}{ LDA } & \multicolumn{2}{|c|}{$\mathrm{PCA}$} \\
\hline Dentr & \multicolumn{2}{|c|}{$76.07 \%$} & $76.29 \%$ & $76.29 \%$ & \multicolumn{2}{|c|}{$76.23 \%$} & \multicolumn{2}{|c|}{$77.02 \%$} \\
\hline $\mathrm{HOF}$ & \multicolumn{2}{|c|}{$85.97 \%$} & $85.73 \%$ & $85.73 \%$ & \multicolumn{2}{|c|}{$86.39 \%$} & \multicolumn{2}{|c|}{$86.15 \%$} \\
\hline $\mathrm{HOG}$ & \multicolumn{2}{|c|}{$84.61 \%$} & $80.97 \%$ & $80.97 \%$ & \multicolumn{2}{|c|}{$85.22 \%$} & \multicolumn{2}{|c|}{$85.02 \%$} \\
\hline $\mathrm{MBH}$ & \multicolumn{2}{|c|}{$88.49 \%$} & $87.48 \%$ & $87.48 \%$ & \multicolumn{2}{|c|}{$88.20 \%$} & \multicolumn{2}{|c|}{$88.94 \%$} \\
\hline place1 fusion & \multicolumn{2}{|c|}{$87.998 \%$} & \multicolumn{2}{|c|}{$86.761 \%$} & \multicolumn{2}{|c|}{$87.80 \%$} & \multicolumn{2}{|c|}{$87.76 \%$} \\
\hline place2 fusion & LDA & PCA & LDA & $\mathrm{PCA}$ & LDA & PCA & LDA & PCA \\
\hline Comb.1 & $\mathbf{9 1 . 4 7 \%}$ & $90.18 \%$ & $90.98 \%$ & $88.76 \%$ & $91.80 \%$ & $91.71 \%$ & $92.25 \%$ & $91.94 \%$ \\
\hline ALL_combine & $91.14 \%$ & $91.14 \%$ & $90.54 \%$ & $88.25 \%$ & $91.56 \%$ & $91.48 \%$ & $90.80 \%$ & $91.23 \%$ \\
\hline place3 fusion $\downarrow$ & \multicolumn{2}{|c|}{ LDA } & \multicolumn{2}{|c|}{$\mathrm{PCA}$} & \multicolumn{2}{|c|}{ LDA } & \multicolumn{2}{|c|}{$\mathrm{PCA}$} \\
\hline product & \multicolumn{2}{|c|}{$90.43 \%$} & \multicolumn{2}{|c|}{$90.04 \%$} & \multicolumn{2}{|c|}{$86.44 \%$} & \multicolumn{2}{|c|}{$87.18 \%$} \\
\hline sum & \multicolumn{2}{|c|}{$90.95 \%$} & \multicolumn{2}{|c|}{$90.05 \%$} & \multicolumn{2}{|c|}{$92.07 \%$} & \multicolumn{2}{|c|}{$\mathbf{9 2 . 5 1} \%$} \\
\hline $\max$ & \multicolumn{2}{|c|}{$90.88 \%$} & \multicolumn{2}{|c|}{$89.31 \%$} & \multicolumn{2}{|c|}{$90.17 \%$} & 90. & $1 \%$ \\
\hline median & 90. & & & $67 \%$ & 90. & $8 \%$ & 91. & $6 \%$ \\
\hline $\min$ & 89. & $0 \%$ & & $86 \%$ & 90. & $9 \%$ & 91. & $5 \%$ \\
\hline major & 89. & & & $91 \%$ & 90 & $4 \%$ & 90. & $2 \%$ \\
\hline
\end{tabular}


Table 3: dimensionality reduction technique comparison on the HMDB51 dataset with different learning methods and fusion methods

\begin{tabular}{|c|c|c|c|c|c|c|c|c|}
\hline learning methods $\rightarrow$ & \multicolumn{4}{|c|}{ SVM } & \multicolumn{4}{|c|}{ ELM } \\
\hline descriptor & \multicolumn{2}{|c|}{ LDA } & \multicolumn{2}{|c|}{ PCA } & \multicolumn{2}{|c|}{ LDA } & \multicolumn{2}{|c|}{ PCA } \\
\hline Dentr & \multicolumn{2}{|c|}{$27.34 \%$} & \multicolumn{2}{|c|}{$37.6 \%$} & \multicolumn{2}{|c|}{$27.39 \%$} & \multicolumn{2}{|c|}{$32.66 \%$} \\
\hline $\mathrm{HOF}$ & \multicolumn{2}{|c|}{$46.06 \%$} & \multicolumn{2}{|c|}{$45.82 \%$} & \multicolumn{2}{|c|}{$46.17 \%$} & \multicolumn{2}{|c|}{$47.17 \%$} \\
\hline $\mathrm{HOG}$ & \multicolumn{2}{|c|}{$36.43 \%$} & \multicolumn{2}{|c|}{$35.90 \%$} & \multicolumn{2}{|c|}{$36.45 \%$} & \multicolumn{2}{|c|}{$37.996 \%$} \\
\hline $\mathrm{MBH}$ & \multicolumn{2}{|c|}{$49.50 \%$} & \multicolumn{2}{|c|}{$45.29 \%$} & \multicolumn{2}{|c|}{$49.13 \%$} & \multicolumn{2}{|c|}{$49.41 \%$} \\
\hline place1 fusion & \multicolumn{2}{|c|}{$22.353 \%$} & \multicolumn{2}{|c|}{$35.21 \%$} & \multicolumn{2}{|c|}{$22.244 \%$} & \multicolumn{2}{|c|}{$40.436 \%$} \\
\hline place2 fusion & LDA & $\mathrm{PCA}$ & LDA & $\mathrm{PCA}$ & LDA & PCA & LDA & PCA \\
\hline Comb.1 & $55.14 \%$ & $55.29 \%$ & $55.32 \%$ & $49.67 \%$ & $54.42 \%$ & $54.42 \%$ & $54.55 \%$ & $55.42 \%$ \\
\hline ALL_combine & $55.80 \%$ & $55.71 \%$ & $\mathbf{5 6 . 2 7} \%$ & $50.57 \%$ & $54.77 \%$ & $54.79 \%$ & $55.84 \%$ & $\mathbf{5 6 . 4 7} \%$ \\
\hline place3 fusion $\downarrow$ & \multicolumn{2}{|c|}{ LDA } & \multicolumn{2}{|c|}{ PCA } & \multicolumn{2}{|c|}{ LDA } & \multicolumn{2}{|c|}{ PCA } \\
\hline product & \multicolumn{2}{|c|}{$55.25 \%$} & \multicolumn{2}{|c|}{$51.74 \%$} & \multicolumn{2}{|c|}{$30.98 \%$} & \multicolumn{2}{|c|}{$53.09 \%$} \\
\hline sum & \multicolumn{2}{|c|}{$54.75 \%$} & \multicolumn{2}{|c|}{$51.24 \%$} & & $9 \%$ & & $3 \%$ \\
\hline $\max$ & & $5 \%$ & 48. & & & $3 \%$ & & $54 \%$ \\
\hline median & & $3 \%$ & 50. & & & $4 \%$ & & $31 \%$ \\
\hline $\min$ & & $9 \%$ & 48. & & & & & $35 \%$ \\
\hline major & & $4 \%$ & 47. & & & $9 \%$ & & $37 \%$ \\
\hline
\end{tabular}


Table 4: dimensionality reduction technique comparison on the UCF101 dataset with different learning methods and fusion methods

\begin{tabular}{|c|c|c|c|c|c|c|c|c|}
\hline learning methods $\rightarrow$ & \multicolumn{4}{|c|}{ SVM } & \multicolumn{4}{|c|}{ ELM } \\
\hline reduction method & \multicolumn{2}{|c|}{ LDA } & \multicolumn{2}{|c|}{ PCA } & \multicolumn{2}{|c|}{ LDA } & \multicolumn{2}{|c|}{ PCA } \\
\hline place1 fusion & \multicolumn{2}{|c|}{$75.549 \%$} & \multicolumn{2}{|c|}{$73.381 \%$} & \multicolumn{2}{|c|}{$80.518 \%$} & \multicolumn{2}{|c|}{$80.782 \%$} \\
\hline place2 fusion & LDA & PCA & LDA & PCA & LDA & PCA & LDA & PCA \\
\hline Comb. 1 & $75.4692 \%$ & $75.76 \%$ & $78.14 \%$ & $76.42 \%$ & $83.72 \%$ & $83.72 \%$ & $84.64 \%$ & $\mathbf{8 4 . 7 7 \%}$ \\
\hline ALL_combine & $75.416 \%$ & $75.839 \%$ & $78.00 \%$ & $75.97 \%$ & $83.69 \%$ & $83.69 \%$ & $83.94 \%$ & $83.98 \%$ \\
\hline place3 fusion $\downarrow$ & \multicolumn{2}{|c|}{ LDA } & \multicolumn{2}{|c|}{$\mathrm{PCA}$} & \multicolumn{2}{|c|}{ LDA } & \multicolumn{2}{|c|}{$\mathrm{PCA}$} \\
\hline product & \multicolumn{2}{|c|}{$75.92 \%$} & \multicolumn{2}{|c|}{$77.06 \%$} & \multicolumn{2}{|c|}{$64.23 \%$} & \multicolumn{2}{|c|}{$81.81 \%$} \\
\hline sum & \multicolumn{2}{|c|}{$76.58 \%$} & \multicolumn{2}{|c|}{$76.95 \%$} & \multicolumn{2}{|c|}{$83.56 \%$} & \multicolumn{2}{|c|}{$83.69 \%$} \\
\hline $\max$ & \multicolumn{2}{|c|}{$76.13 \%$} & \multicolumn{2}{|c|}{$76.02 \%$} & \multicolumn{2}{|c|}{$76.34 \%$} & \multicolumn{2}{|c|}{$78.80 \%$} \\
\hline median & \multicolumn{2}{|c|}{$74.73 \%$} & \multicolumn{2}{|c|}{$75.63 \%$} & \multicolumn{2}{|c|}{$79.80 \%$} & \multicolumn{2}{|c|}{$81.87 \%$} \\
\hline $\min$ & \multicolumn{2}{|c|}{$68.78 \%$} & \multicolumn{2}{|c|}{$73.80 \%$} & \multicolumn{2}{|c|}{$76.66 \%$} & \multicolumn{2}{|c|}{$78.69 \%$} \\
\hline major & \multicolumn{2}{|c|}{$73.57 \%$} & \multicolumn{2}{|c|}{$74.14 \%$} & \multicolumn{2}{|c|}{$79.01 \%$} & \multicolumn{2}{|c|}{$79.46 \%$} \\
\hline
\end{tabular}

On the other hand, "red and green bar" of Fig.5 shows that LDA with ELM obtains weaker performance than PCA with ELM. From "yellow and red bar" of Fig.5, it obviously shows that LDA with SVM achieved the near perfect performance compared with the LDA with ELM. Seen from "green and blue bar" of Fig.5, PCA with ELM obtains better performance than PCA with SVM except the Dentr's FV of HMDB51 and HOF's FV of KTH. Interestingly, ignoring the special case such as Dentr's FV of HMDB51, it obviously shows that PCA with SVM has the worst result while PCA with ELM has the best performance in terms of mAP and time. In addition, LDA with ELM is equal to LDA with SVM. From the experiments, it can be seen that LDA can save more memory space and classify faster than PCA, shown in table 7. In table 7, since ELM is faster than SVM, it's not obvious for PCA and LDA in terms of times. Therefore, we use SVM's time to compare. In addition, HMDB51 is used to compare the efficiency of LDA and PCA as it is more obvious for time. Table 7 shows 
testing.

\subsection{The performance of different fusion methods}

In this section, we mainly analyze the impact of different fusion methods. Table 1-4 demonstrate that place1 fusion can slightly improve the performance than that ELM for all kinds of situations in KTH dataset. By analyzing table 2, it indicates that ELM is more comparable to or better than SVM in UCF11. The best mAP of ELM is up to $1 \%$ which is better than SVM. What's more, 
ELM saves a lot of time due to that it does not needs to be tuned parameters, basketball shooting and tennis swinging.

Fig. 8 shows the best confusion matrix of heat map of HMDB51. Numbers are not clear. The color legend is drawn at the right (please view in color). marked in table 5. It shows that the per-accuracy of pick, smile, swing baseball, sword exercise, throw and wave are all less than 20\%. Smile is confused with chew, laugh and talk. It is obvious to show that actions are confused with each other which being in the same group. Sword exercise is most confused 
shows that the most low accuracy situation is the type of body movements with object interaction. In the future, we will consider how to distinguish the actions which are in the same group and explore how to distinguish the action of body movements with object interaction. In the subsection, we will compare our best

\subsection{Compare to the stat-of-the-art}

In order to quantify the effectiveness of the method, the obtained results are compared qualitatively with those obtained by other best known state-of-the art methods (shown table 9). It shows that our descriptor has a classification accuracy of $97.22 \%$, which is comparable to the state of the art. As far as we know, the best accuracy for $\mathrm{KTH}$ is $98.2 \%$ which is obtained by a high-level representation [43] and it requires non-trivial amounts of hand-labeled training data per each semantic entity (object, part, attribute, etc). Therefore, our method is the best one for the low-level representation on KTH dataset. For 355 a fair comparison, we list Peng et al. 44]'s mAP with traditional FV and stacked FV, respectively. What's more, Wang et al.[3]'s mAP is separately listed with \without human detection (HD). Table 9 can be seen that on the UCF11 dataset, our method improved by $2 \%$ with the same extracting features method, without preprocessing (e.g. HD). The best result of UCF101 is $84.77 \%$ which

\section{Conclusion}

This paper is to evaluate the effects of dimensionality reduction, fusion methods and learning methods for human action recognition. We find that PCA with 


\begin{tabular}{|c|c|c|c|c|c|c|}
\hline boxing & 1.00 & 0.00 & 0.00 & 0.00 & 0.00 & 0.00 \\
\hline handclapping & 0.00 & 1.00 & 0.00 & 0.00 & 0.00 & 0.00 \\
\hline handwaving & 0.00 & 0.06 & 0.94 & 0.00 & 0.00 & 0.00 \\
\hline jogging & 0.00 & 0.00 & 0.00 & 1.00 & 0.00 & 0.00 \\
\hline running & 0.00 & 0.00 & 0.00 & 0.11 & 0.89 & 0.00 \\
\hline walking & 0.00 & 0.00 & 0.00 & 0.00 & 0.00 & 1.00 \\
\hline
\end{tabular}

Figure 6: The best confusion matrix for KTH

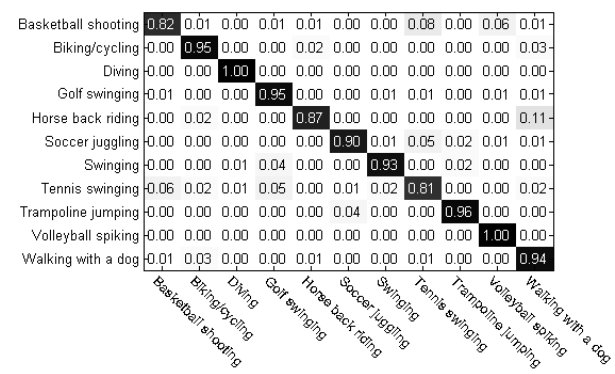

Figure 7: The best confusion matrix for UCF11

ELM has a better result and the place2 fusion has a stable or better result compared to place1 and place3 fusion. While SVM has no obvious character about which dimensionality reduction techniques should be used with $100 \%$ power rate. In future work is to learn which power rate is best for recognition rate. We also analyze the cost of the training time and testing time. It shows that our method can improve accuracy and compute efficiency. Finally, we achieve the state-of-the-art results on UCF11 and HMDB51 datasets by applying our

best configuration with the same conditions. 


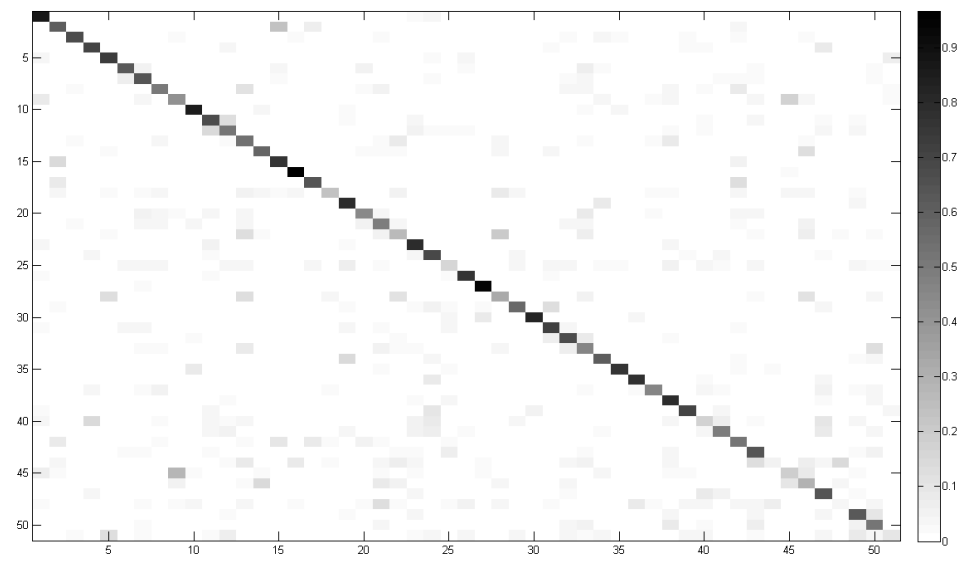

Figure 8: The heat map of best confusion matrix for HMDB51

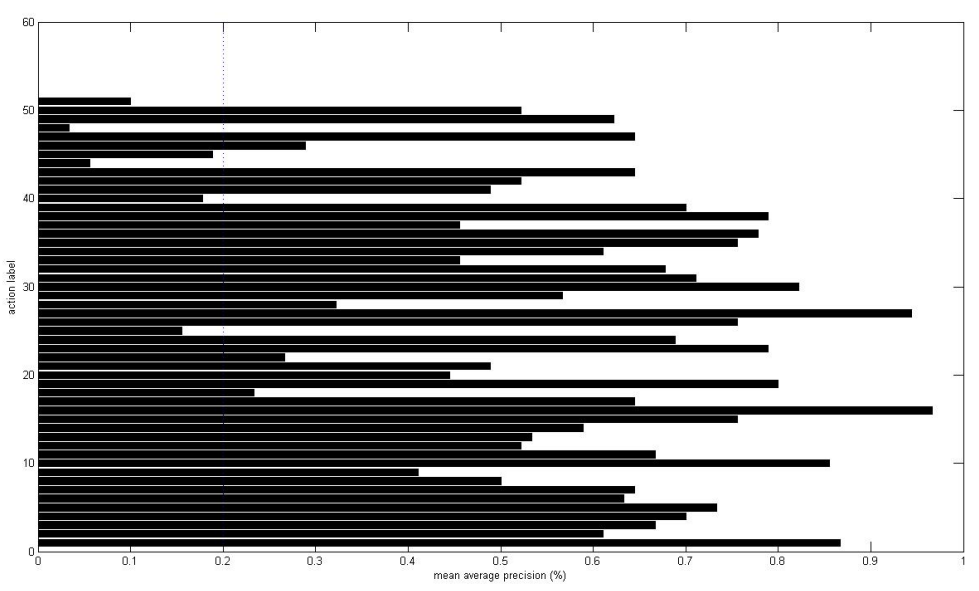

Figure 9: The bar figure of per class mAP for HMDB51 
Table 5: Per class (sorted) mAP for HMDB51

\begin{tabular}{|c|c|c|c|}
\hline 1:brush_hair 86.67\% & 14:fencing $58.89 \%$ & 27:pullup $94.44 \%$ & 40: smile $17.78 \%$ \\
\hline 2:cartwheel $61.11 \%$ & 15:flic_flac $75.56 \%$ & 28:punch $32.22 \%$ & 41:smoke $48.89 \%$ \\
\hline 3:catch $66.67 \%$ & 16 :golf $96.67 \%$ & 29:push $56.67 \%$ & 42:somesault $52.22 \%$ \\
\hline 4:chew $70.00 \%$ & 17 :handstand $64.44 \%$ & 30:pushup $82.22 \%$ & $43:$ stand $64.44 \%$ \\
\hline 5:clap $73.33 \%$ & 18:hit $23.33 \%$ & 31:ride_bike $71.11 \%$ & 44:swing_baseball $5.56 \%$ \\
\hline 6:climb $63.33 \%$ & 19:hug $80.00 \%$ & 32:ride_horse $67.78 \%$ & 45 :sword_exercise $18.89 \%$ \\
\hline 7:climb_stairs $64.44 \%$ & $20:$ jump $44.44 \%$ & 33 :run $45.56 \%$ & 46 :sword $28.89 \%$ \\
\hline 8:dive $50.00 \%$ & 21:kick_ball $48.89 \%$ & 34:shake_hands $61.11 \%$ & 47 :talk $64.44 \%$ \\
\hline 9:draw_sword $41.11 \%$ & 22:kick $26.67 \%$ & 35:shoot_ball $75.56 \%$ & 48:throw $3.33 \%$ \\
\hline 10:dribble $85.56 \%$ & $23:$ kiss $78.89 \%$ & 36:shoot_bow $77.78 \%$ & 49:turn $62.22 \%$ \\
\hline 11:drink $66.67 \%$ & 24:laugh $68.89 \%$ & 37:shoot_gun $45.56 \%$ & 50:walk $52.22 \%$ \\
\hline 12:eat $52.22 \%$ & 25:pick $15.56 \%$ & $38:$ sit $78.89 \%$ & 51:wave $10.00 \%$ \\
\hline 13:fall_floor $53.33 \%$ & 26:pour $75.56 \%$ & $39:$ situp $70.00 \%$ & \\
\hline
\end{tabular}

Table 6: pca \lda and non_pca comparison in terms of time for SVM on UCF11

\begin{tabular}{|c|c|c|c|c|c|c|}
\hline & \multicolumn{2}{|c|}{ LDA } & \multicolumn{2}{c|}{ PCA } & \multicolumn{2}{c|}{ non_PCA } \\
\hline descriptor & tr_time(s) & te_time(s) & tr_time(s) & te_time(s) & tr_time(s) & te_time(s) \\
\hline Dentr & 0.0500 & 0.0759 & 14.2178 & 0.4320 & 151.0652 & 1.9528 \\
HOF & 0.0494 & 0.1359 & 15.4737 & 0.5786 & 522.8764 & 4.1085 \\
HOG & 0.0535 & 0.0715 & 16.9994 & 0.9035 & 584.5440 & 4.9395 \\
MBH & 0.0630 & 0.0161 & 16.3682 & 0.5582 & 1314.2 & 10.8843 \\
\hline
\end{tabular}

Table 7: LDA and PCA comparison in terms of time with the place2 fusion for SVM on HMDB51

\begin{tabular}{|c|c|c|}
\hline & $\mathrm{PCA}(\mathrm{s})$ & $\mathrm{LDA}(\mathrm{s})$ \\
\hline training time & 787.79 & 0.78 \\
\hline testing time & 32.86 & 0.33 \\
\hline
\end{tabular}


Table 8: ELM and SVM comparison in terms of time with the place1 fusion on HMDB51

\begin{tabular}{|c|c|c|c|}
\hline & & $\mathrm{SVM}(\mathrm{s})$ & $\mathrm{ELM}(\mathrm{s})$ \\
\hline \multirow{2}{*}{$\mathrm{PCA}$} & training time & 415.17 & 2.69 \\
\cline { 2 - 4 } & testing time & 34.09 & 1.37 \\
\hline \hline \multirow{2}{*}{ LDA } & training time & 8.26 & 0.85 \\
\cline { 2 - 4 } & testing time & 1.67 & 0.071 \\
\hline
\end{tabular}

\section{Acknowledgment}

This work was partly supported by the National Science Foundation of China (Grant No.61203251 and No.61574035) and Key Foundation of Jiangsu (Grant No.BK2011018), the Fundamental Research Funds for the Central Universities and Graduate Research and Innovation Projects of Universities in Jiangsu Province (KYLX_0129), the Fundamental Research Funds of Southeast University (SJLX_0074)

\section{References}

[1] H. Wang, M. M. Ullah, A. Klaser, I. Laptev, C. Schmid, Evaluation of local spatio-temporal features for action recognition, in: BMVC 2009-British Machine Vision Conference.

[2] H. Wang, A. Klaser, C. Schmid, C.-L. Liu, Action recognition by dense trajectories, in: Computer Vision and Pattern Recognition (CVPR), 2011 IEEE Conference on, IEEE, pp. 3169-3176.

[3] H. Wang, C. Schmid, Action recognition with improved trajectories, in: International Conference on Computer Vision, 2013.

[4] H. Wang, A. Klaser, C. Schmid, C.-L. Liu, Dense trajectories and motion boundary descriptors for action recognition, International Journal of Computer Vision (2013) 1-20. 
Table 9: Comparison of our method with state-of-the-art methods in the literature

\begin{tabular}{|c|c|c|}
\hline datasets & methods & recognition rate \\
\hline \multirow{6}{*}{$\mathrm{KTH}$} & Sadanand et al. 43 & $98.2 \%$ \\
\hline & ourmethod & $97.22 \%$ \\
\hline & Wang et al. 4 & $95.0 \%$ \\
\hline & $\mathrm{Wu}$ et al. 38 & $94.5 \%$ \\
\hline & Bilinski et al. 47 ] & $96.16 \%$ \\
\hline & Wang et al. 48 & $93.3 \%$ \\
\hline \multirow{7}{*}{ UCF11 } & Peng et al. 44. (stacked FV) & $93.38 \%$ \\
\hline & ourmethod & $92.51 \%$ \\
\hline & Wang et al. 4 & $85.4 \%$ \\
\hline & Peng et al. 44 & $90.69 \%$ \\
\hline & Zhe et al. 49 & $89.4 \%$ \\
\hline & Sapienza et al. 50 & $87.2 \%$ \\
\hline & Liu et al. 40 & $71.2 \%$ \\
\hline \multirow{9}{*}{ HMDB51 } & Fernando et al. 46] (VideoDarwin) & $63.7 \%$ \\
\hline & Peng et al. 44 (stacked FV) & $66.79 \%$ \\
\hline & Wang et al. 3] (HD) & $57.2 \%$ \\
\hline & ourmethod & $56.47 \%$ \\
\hline & Wang et al. 4 & $48.3 \%$ \\
\hline & Wang et al. 3] & $55.9 \%$ \\
\hline & Zhe et al. 49 & $54 \%$ \\
\hline & Sapienza et al. 50] & $40.7 \%$ \\
\hline & Wang et al. 48 & $42.1 \%$ \\
\hline
\end{tabular}


[11] H. Jgou, M. Douze, C. Schmid, P. Prez, Aggregating local descriptors into a compact image representation, in: Computer Vision and Pattern Recognition (CVPR), 2010 IEEE Conference on, IEEE, pp. 3304-3311.

[12] M. Jain, H. Jgou, P. Bouthemy, Better exploiting motion for better action recognition, in: CVPR-International Conference on Computer Vision and Pattern Recognition, 2013.

[13] J. Liu, J. Luo, M. Shah, Recognizing realistic actions from videos in the wild, in: Computer Vision and Pattern Recognition, 2009. CVPR 2009. IEEE Conference on, IEEE, pp. 1996-2003. tion recognition, in: IEEE Intenational Conference on Computer Vision Workshops (ICCV2013), Vol. 4321.

[6] H. Xu, Q. Tian, Z. Wang, J. Wu, A survey on aggregating methods for action recognition with dense trajectories, in: Multimedia tools and appli-

[7] M. M. Ullah, S. N. Parizi, I. Laptev, Improving bag-of-features action recognition with non-local cues, in: BMVC, Vol. 10, 2010, pp. 95.1-95.11.

[8] J. Yu, M. Jeon, W. Pedrycz, Weighted feature trajectories and concatenated bag-of-features for action recognition, Neurocomputing 131 (2014) 200-207.

9] A.-A. Liu, N. Xu, Y.-T. Su, H. Lin, T. Hao, Z.-X. Yang, Single/multi-view human action recognition via regularized multi-task learning, Neurocomputing 151 (2015) 544-553.

[10] F. Perronnin, J. Snchez, T. Mensink, Improving the fisher kernel for largescale image classification, Springer, 2010, pp. 143-156.

Z.-z. Lan, L. Bao, S.-I. Yu, W. Liu, A. G. Hauptmann, Multimedia clas-

] O. R. Murthy, R. Goecke, Ordered trajectories for large scale human acsification and event detection using double fusion, Multimedia Tools and Applications (2013) 1-15. 
[15] M. Marszalek, I. Laptev, C. Schmid, Actions in context, in: Computer Vision and Pattern Recognition, 2009. CVPR 2009. IEEE Conference on, IEEE, pp. 2929-2936.

[16] Z. Cai, L. Wang, X. Peng, Y. Qiao, Multi-view super vector for action recognition, in: Computer Vision and Pattern Recognition, 2014. CVPR 2014. IEEE Conference on.

[17] A. Iosifidis, A. Tefas, I. Pitas, Regularized extreme learning machine for multi-view semi-supervised action recognition, Neurocomputing 145 (2014) 250-262.

[18] R. Minhas, A. Baradarani, S. Seifzadeh, Q. Jonathan Wu, Human action recognition using extreme learning machine based on visual vocabularies, Neurocomputing 73 (10) (2010) 1906-1917.

${ }_{435}$ [19] G. Varol, A. A. Salah, Extreme learning machine for large-scale action recognition, in: ECCV workshop, Vol. 7, p. 8.

[20] D. Oneata, J. Verbeek, C. Schmid, Action and event recognition with fisher vectors on a compact feature set, in: Computer Vision (ICCV), 2013 IEEE International Conference on, IEEE, pp. 1817-1824.

[21] S. Karaman, L. Seidenari, S. Ma, A. Del Bimbo, S. Sclaroff, Adaptive structured pooling for action recognition, in: Proc. of British Machine Vision Conference (BMVC).

[22] F. Shi, Local part model for action recognition in realistic videos, Thesis (2014).

${ }_{445}$ [23] F. Shi, R. Laganiere, E. Petriu, Lpm for action recognition in temporally untrimmed videos.

[24] N. Ikizler, R. G. Cinbis, S. Pehlivan, P. Duygulu, Recognizing actions from still images, in: Pattern Recognition, 2008. ICPR 2008. 19th International Conference on, IEEE, pp. 1-4. 
[25] D. Cai, X. He, J. Han, Srda: An efficient algorithm for large-scale discriminant analysis, Knowledge and Data Engineering, IEEE Transactions on 20 (1) (2008) 1-12.

[26] H. Kuehne, H. Jhuang, E. Garrote, T. Poggio, T. Serre, Hmdb: a large video database for human motion recognition, in: Computer Vision (ICCV), 2011 IEEE International Conference on, IEEE, pp. 2556-2563.

[27] K. Soomro, A. R. Zamir, M. Shah, Ucf101: A dataset of 101 human actions classes from videos in the wild, arXiv preprint arXiv:1212.0402.

[28] O. R. Murthy, R. Goecke, Combined ordered and improved trajectories for large scale human action recognition, in: 2013 ICCV Workshop on Action Recognition with a Large Number of Classes.

[29] H. Xu, Q. Tian, Z. Wang, J. Wu, Human action recognition using late fusion and dimensionality reduction, in: Digital Signal Processing (DSP), 2014 19th International Conference on, IEEE, pp. 63-67.

[30] H. Jgou, F. Perronnin, M. Douze, C. Schmid, Aggregating local image descriptors into compact codes, Pattern Analysis and Machine Intelligence, IEEE Transactions on 34 (9) (2012) 1704-1716.

[31] J. Liu, S. Ali, M. Shah, Recognizing human actions using multiple features, in: Computer Vision and Pattern Recognition, 2008. CVPR 2008. IEEE Conference on, IEEE, pp. 1-8.

[32] I. Laptev, M. Marszalek, C. Schmid, B. Rozenfeld, Learning realistic human actions from movies, in: Computer Vision and Pattern Recognition, 2008. CVPR 2008. IEEE Conference on, IEEE, pp. 1-8.

[33] M. Bregonzio, J. Li, S. Gong, T. Xiang, Discriminative topics modelling for action feature selection and recognition, in: BMVC, 2010, pp. 1-11.

[34] D. Zhao, L. Shao, X. Zhen, Y. Liu, Combining appearance and structural features for human action recognition, Neurocomputing 113 (2013) 88-96. 
[35] Z. Cheng, L. Qin, Q. Huang, S. Yan, Q. Tian, Recognizing human group action by layered model with multiple cues, Neurocomputing 136 (2014) $124-135$.

[36] G.-B. Huang, Q.-Y. Zhu, C.-K. Siew, Extreme learning machine: theory and applications, Neurocomputing 70 (1) (2006) 489-501.

[37] G.-B. Huang, H. Zhou, X. Ding, R. Zhang, Extreme learning machine for regression and multiclass classification, Systems, Man, and Cybernetics, Part B: Cybernetics, IEEE Transactions on 42 (2) (2012) 513-529.

[38] X. Wu, D. Xu, L. Duan, J. Luo, Action recognition using context and appearance distribution features, in: Computer Vision and Pattern Recognition (CVPR), 2011 IEEE Conference on, IEEE, pp. 489-496.

[39] C. Schuldt, I. Laptev, B. Caputo, Recognizing human actions: a local svm approach, in: Pattern Recognition, 2004. ICPR 2004. Proceedings of the 17th International Conference on, Vol. 3, IEEE, pp. 32-36.

[40] J. Liu, J. Luo, M. Shah, Recognizing realistic actions from videos in the wild, in: Computer Vision and Pattern Recognition, 2009. CVPR 2009. IEEE Conference on, IEEE, pp. 1996-2003.

[41] H. Xu, Q. Tian, J. Wu, Exploring the influence of motion boundary sampling to improved dense trajectories for action recognition, in: Proceedings of the 7th International Conference on Internet Multimedia Computing and Service, ACM, p. 62 .

[42] G.-B. Huang, D. H. Wang, Y. Lan, Extreme learning machines: a survey, International Journal of Machine Learning and Cybernetics 2 (2) (2011) $107-122$.

[43] S. Sadanand, J. J. Corso, Action bank: A high-level representation of activity in video, in: Computer Vision and Pattern Recognition (CVPR), 2012 IEEE Conference on, IEEE, pp. 1234-1241. 
[44] X. Peng, C. Zou, Y. Qiao, Q. Peng, Action Recognition with Stacked Fisher Vectors, Springer, 2014, pp. 581-595.

[45] http://crcv.ucf.edu/iccv13-action-workshop/.

[46] O. J. e. a. Fernando B, Gavves E, Modeling video evolution for action recognition, in: Computer Vision and Pattern Recognition, 2015. CVPR 2015. IEEE Conference on, IEEE.

[47] P. Bilinski, F. Bremond, Contextual statistics of space-time ordered features for human action recognition, in: Advanced Video and Signal-Based Surveillance (AVSS), 2012 IEEE Ninth International Conference on, IEEE, pp. 228-233.

[48] L. Wang, Y. Qiao, X. Tang, Motionlets: Mid-level 3d parts for human motion recognition, in: Computer Vision and Pattern Recognition (CVPR), 2013 IEEE Conference on, IEEE, pp. 2674-2681.

[49] J. Zhu, B. Wang, X. Yang, W. Zhang, Z. Tu, Action recognition with actons, in: Computer Vision (ICCV), 2013 IEEE International Conference on, IEEE, pp. 3559-3566.

${ }_{520}$ [50] M. Sapienza, F. Cuzzolin, P. H. Torr, Learning discriminative spacectime action parts from weakly labelled videos, International Journal of Computer Vision (2014) 1-18. 